High Energy Phenomena in Relativistic Outflows (HEPRO IV) International Journal of Modern Physics: Conference Series Vol. 28 (2014) 1460182 (6 pages)

(C) The Authors

DOI: $10.1142 / \mathrm{S} 2010194514601823$

\title{
ON THE GAMMA-RAY EMISSION FROM THE CORE AND RADIO LOBES OF THE RADIO GALAXY CENTAURUS A*
}

\author{
NAREK SAHAKYAN ${ }^{\dagger}$,, FRANK M. RIEGER ${ }^{\ddagger}$ and FELIX AHARONIAN A $^{\ddagger}$ \\ ${ }^{\dagger}$ ICRANet-Yerevan, Marshall Baghramian Avenue, 24, Yerevan 0019, Republic of Armenia \\ and \\ ICRANet, Piazza della Repubblica 10, I-65122 Pescara, Italy \\ ${ }^{\ddagger}$ Max-Planck-Institut für Kernphysik, P.O. Box 103980, 69029 Heidelberg, Germany \\ $\S$ Dublin Institute for Advanced Studies, 31 Fitzwilliam Place, Dublin 2, Ireland \\ Tnarek@icra.it \\ RUIZHI YANG $\|$ and EMMA DE ONA-WILHELMI**

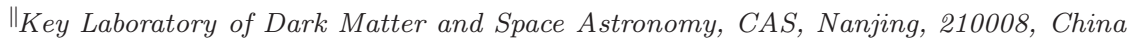 \\ **Institut de Cincies de l'Espai (IEEC-CSIC), Barcelona 08193, Spain
}

Received 31 October 2013

Revised 5 December 2013

Published 21 March 2014

\begin{abstract}
We summarize recent results based on an analysis of Fermi-LAT data for the lobes and the core of the nearby radio galaxy Centaurus A (Cen A). In the case of the core, high-energy $(\mathrm{HE} ;>100 \mathrm{MeV}) \gamma$-rays up to $50 \mathrm{GeV}$ have been detected with a detection significance of about $44 \sigma$. The average gamma-ray spectrum of the core reveals interesting evidence for a possible deviation from a simple power-law. A likelihood analysis with a broken powerlaw model shows that the photon index becomes substantially harder above $E_{b} \simeq 4 \mathrm{GeV}$, changing from $\Gamma_{1}=2.74 \pm 0.03$ below to $\Gamma_{2}=2.09 \pm 0.20$ above. It seems possible that this hardening marks the contribution of an additional high-energy component beyond the common synchrotron-self Compton jet emission.

In the case of the lobes, the high-energy gamma-ray emission extends up to $6 \mathrm{GeV}$, with a significance of more than 10 and $20 \sigma$ for the north and the south lobe, respectively. Based on a detailed spatial analysis and comparison with the associated radio lobes, a substantial extension of the HE $\gamma$-ray emission beyond the WMAP radio image for the northern lobe of Cen A is found. We provide a short discussion of the lobe's spectral energy distribution (SED) in the context of hadronic and time-dependent leptonic scenarios.
\end{abstract}

Keywords: Galaxies: active; galaxies: Cen A, gamma rays, radiation mechanisms: nonthermal.

PACS Numbers: 98.54.Gr, 95.85.Pw, 95.30.Gv

*This is an Open Access article published by World Scientific Publishing Company. It is distributed under the terms of the Creative Commons Attribution 3.0 (CC-BY) License. Further distribution of this work is permitted, provided the original work is properly cited. 


\section{Introduction}

The prominent radio galaxy Centaurus A (NGC 5128), at a distance of $\simeq 3.8$ $\mathrm{Mpc},{ }^{1}$ is our closest active galaxy. Often considered as a prototype Fanaroff-Riley Class $\mathrm{I}^{2}$ radio source and as a misaligned BL Lac-type object at higher energies, ${ }^{3,4}$ it is one of the best-studied extragalactic objects over a wide range of frequencies. $^{5}$ Observations at radio frequencies show giant structures (so-called 'lobes') with a total angular size of $\sim 10^{\circ}$, which corresponds to a physical extension of $\sim 600 \mathrm{kpc}(\mathrm{d} / 3.8 \mathrm{Mpc})$.

The Fermi-LAT collaboration has early on reported the detection of HE $\gamma$-rays from both the core (i.e., within $\sim 0.1^{\circ}$ ) and the giant radio lobes of Cen A:6,7 An analysis of the available ten-month LAT data set revealed a point-like HE emission region coincident with the position of the radio core of $\mathrm{Cen} \mathrm{A}$, and two large extended emission regions detected with a significance of 5 and $8 \sigma$ for the northern and the southern lobe, respectively. On the other hand, the HE emission from the extended regions seemed to be morphologically correlated with the giant radio lobes, contributing more than $50 \%$ to the total HE source emission. These regions appeared spectrally well described by a power-law function extending up to 2 or 3 $\mathrm{GeV}$ with photon indices of $\Gamma \sim 2.6$.

Here we report on our results obtained using a much larger Fermi-LAT data set, allowing for a detailed investigation of the spectrum and morphology of the 'lobes' and for an improved analysis of the spectral and temporal characteristics of $\mathrm{HE}$ core emission.

\section{Fermi-LAT Data Analysis}

Here we present the main observational results of a Fermi-LAT analysis of HE core and lobe emission in Cen A. Detailed information on the analysis can be found in Refs. 8, 9 .

\subsection{The core of Cen $A$}

The continuum gamma-ray emission of the core of Cen A has first been modeled with a single power law and a binned gtlike analysis been performed. The best-fit power-law parameters for the core of Cen A turned out to be

$$
\left(\frac{d N}{d E}\right)_{P}=(2.73 \pm 0.12) \times 10^{-9}\left(\frac{E}{100 \mathrm{MeV}}\right)^{-2.69 \pm 0.03}
$$

The test statistic is $T S=1978$ above $100 \mathrm{MeV}$, corresponding to a $\approx 44 \sigma$ detection significance. Figure 1(a) shows the spectrum of the core of Cen A obtained by separately running gtlike for 12 energy bands, where the dashed line shows the bestfit single power-law function for the data given in Eq. (1). For the highest energy bin (56.2-100 GeV), an upper limit is shown. A closer inspection shows, however, 


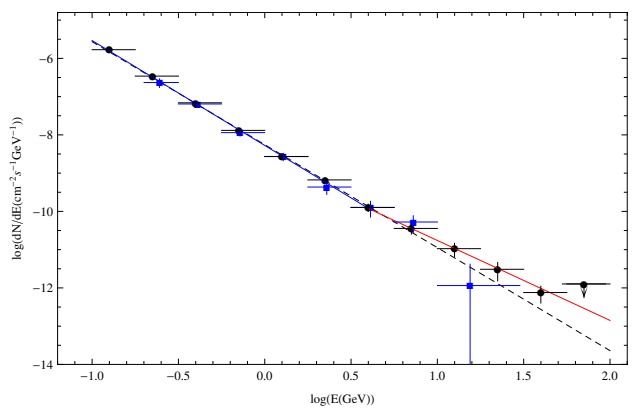

(a)

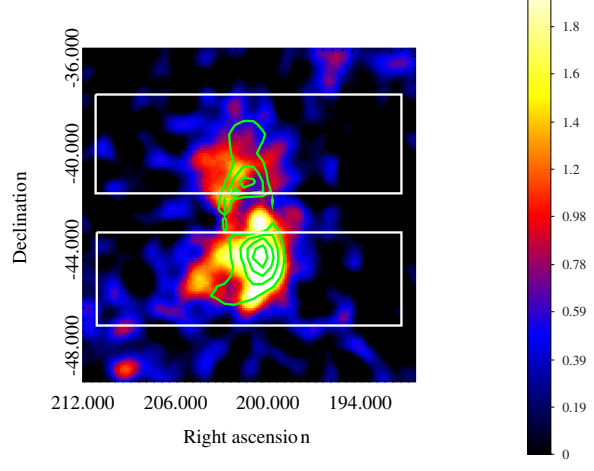

(b)

Fig. 1. (a) Average high-energy gamma-ray $(>100 \mathrm{MeV})$ spectrum of the core of Cen A. The power-law function from the Eq. (1) is depicted with dashed black line. (b) Cen A 'lobes': Excess map after subtraction of diffuse background, point-like sources and Cen A core.

that the measured spectrum exhibits clear deviations from a single power-law model with respect to the data above several $\mathrm{GeV}\left(\chi^{2}\right.$ fit of the power-law model to the data gives a relatively poor fit with $\chi^{2}=39.7$ for 9 dof). In order to analyze this in more detail, the core spectrum has been modeled with a broken power-law model and gtlike tool retried. The best-fit broken power-law parameters are now

$$
\left(\frac{d N}{d E}\right)_{B P}=(1.19 \pm 0.08) \times 10^{-13}\left(\frac{E}{E_{b}}\right)^{-\Gamma_{1,2}}
$$

with $\Gamma_{1}=2.74 \pm 0.02$ and $\Gamma_{2}=2.12 \pm 0.14$ below and above $E_{b}=(4.00 \pm 0.09) \mathrm{GeV}$, respectively. The power-law and the broken-power-law models can be compared using a log likelihood ratio test. The test statistic is twice the difference in these loglikelihoods, which gives 9 for this case. Note that the probability distribution of the test statistic can be approximated by a $\chi^{2}$ distribution with 2 dof, corresponding to different degrees of freedom between the two functions. In this case $P\left(\chi^{2}\right)=0.011$, which again indicates a deviation from a simple power-law function.

The results presented above indicate an interesting hardening of the (average) gamma-ray core spectrum towards higher energies. The break in the spectrum at $4 \mathrm{GeV}$ could most naturally be explained by a superposition of different spectral components. If one divides the data set into two energy ranges, i.e., [0.1-4] GeV and [4-100] GeV, modelling each energy range separately with a power-law function, the analysis gives a photon index of $\Gamma_{1}=2.74 \pm 0.02$ and a flux $F_{\gamma}=(1.68 \pm 0.04) \times 10^{-7}$ photon $\mathrm{cm}^{-2} \mathrm{~S}^{-1}$ for the $[0.1-4] \mathrm{GeV}$ interval (test statistics gives $\mathrm{TS}=1944$ ) and $\Gamma_{2}=2.09 \pm 0.2$, and $F_{\gamma}=(4.20 \pm 0.64) \times 10^{-10}$ photon $\mathrm{cm}^{-2} \mathrm{~s}^{-1}$ for the $[4-100]$ $\mathrm{GeV}$ range (TS value 124.4), respectively. These components are depicted with a blue and red line in Fig. 1(a). 


\subsection{The lobes of Cen $A$}

For the analysis of Fermi-LAT data of the lobes events with energies between 200 $\mathrm{MeV}$ and $30 \mathrm{GeV}$ were selected. Figure 1(b) shows the residual image obtained after subtracting point like sources (including the core of Cen A) and the diffuse background. In order to evaluate the total (extended) HE $\gamma$-ray emission in more detail, a template based on the residual map (T1) has been used. The TS values for the south and the north lobe in this template are 411 and 155, respectively. Green contours in Fig. 1(b) show the radio lobes (WMAP, 22 GHz), with the higherfrequency $22 \mathrm{GHz}$ map being used as it better represents the $\mathrm{GeV}$-emitting particles. A closer inspections reveals that the south lobe of the HE $\gamma$-ray image is roughly similar to the south lobe of the radio one, whereas the HE emission in the north seems to extend beyond the radio lobe emission region Fig. 1(b). A morphological analysis in fact indicates some incongruity between the morphology of the radio lobe and $\gamma$-ray lobe in the north. This is particularly instructive as HE gammarays directly trace the underlying spatial distribution of energetic electrons (via IC processes) without the degeneracy involved in the radio synchrotron part (i.e., uncertainties in magnetic field topology). Using the template generated with the residual map (T1), the total flux and photon index of the north and the south lobe is derived for the energy range from $100 \mathrm{MeV}$ to $30 \mathrm{GeV}$. For the north lobe, the integral HE flux is $(0.93 \pm 0.09) \times 10^{-7} \mathrm{ph} \mathrm{cm}^{-2} \mathrm{~s}^{-1}$ and the photon index is $2.24 \pm 0.08$, while for the south lobe we find $(1.4 \pm 0.2) \times 10^{-7} \mathrm{ph} \mathrm{cm}^{-2} \mathrm{~s}^{-1}$ and $2.57 \pm 0.07$, respectively.

\section{Discussion}

The core of Cen A: The analysis of the 4 yr-data set interestingly reveals that the $\mathrm{HE}$ core spectrum of Cen A shows an unusual break, with photon index changing from $\simeq 2.7$ to $\simeq 2.1$ at an energy of $E_{b} \simeq 4 \mathrm{GeV}$. While spectral breaks are often associated with a situation where the spectrum gets softer, here the opposite occurs. For the component below $4 \mathrm{GeV}$, the detected photon flux $F_{\gamma}=(1.68 \pm 0.04) \times$ $10^{-7}$ photon $\mathrm{cm}^{-2} \mathrm{~s}^{-1}$ corresponds to an apparent (isotropic) $\gamma$-ray luminosity of $L_{\gamma}(0.1-4 \mathrm{GeV}) \simeq 10^{41} \mathrm{erg} \mathrm{s}^{-1}$ (for the distance $3.8 \mathrm{Mpc}$ ). On the other hand, the HE luminosity of the component above $4 \mathrm{GeV}$ translates into $L_{\gamma}(>4 \mathrm{GeV}) \simeq$ $1.4 \times 10^{40} \mathrm{erg} \mathrm{s}^{-1}$. This is an order of magnitude less when compared with the first component, but still larger than the VHE luminosity reported by H.E.S.S. $L_{\gamma}(>250 \mathrm{GeV})=2.6 \times 10^{39} \mathrm{erg} \mathrm{s}^{-1} \cdot{ }^{10}$ In fact, the spectral hardening seen at $\mathrm{GeV}$ energies may allow to account for the (non-simultaneous) H.E.S.S. flux data.

The limited angular resolution $(\sim 5 \mathrm{kpc})$ and the lack of significant variability introduces substantial uncertainties as to the production site of the HE gammaray emission. In principle, the hard $\mathrm{HE}$ component could originate from both a very compact (sub-pc) and/or extended (multi-kpc) region(s). The double-peaked nuclear SED of Cen A has in the past been reasonably well-modeled with simple one zone SSC models up to a few $\mathrm{GeV} .{ }^{4,7}$ In this framework, the observed break at 
$4 \mathrm{GeV}$ would indeed mark the appearance of a physically different component. This additional component could in principle be related to a number of different (not mutually exclusive) scenarios, from magnetosphere VHE emission ${ }^{11}$ up to inverse Compton processes in the kpc-scale jet, ${ }^{12}$ see Ref. 9 for a full discussion. At the current stage, none of these models can be easily discarded. Definite progress concerning the true origin of the HE gamma-ray could be achieved, however, in case of a significant detection of gamma-ray time variability.

The lobes of Cen A: The HE $\gamma$-ray emission observed from the lobes could in principle be related to leptonic (inverse-Compton scattering) or hadronic (e.g., ppinteraction) processes. In the following, we briefly summarize possible constraints for the underlying radiation mechanism imposed by the observed SEDs (for detailed information see Ref. 8). In a leptonic scenario, both the HE $\gamma$-ray and the radio emission could be satisfactorily accounted for. Assuming an electron injection spectrum with an exponential cut-off, the emergent electron distribution, found by solving the kinetic equation describing the energetic and temporal evolution of the radiating electrons, can been used to model the SED through synchrotron and inverseCompton emission.

The duration of particle acceleration (associated with this the age of the giant lobe emission) is unknown. Dynamical arguments suggest a lower limit $>10^{7} \mathrm{yr}$ for the giant radio lobes, while synchrotron spectral ageing arguments indicate an age $\lesssim 3 \times 10^{7}$ yr. $^{5,13,14}$ In the following, we therefore discuss the SED implications for an epoch time $t$ between $10^{7} \mathrm{yr}$ and $10^{8} \mathrm{yr}$. As it turns out, the modeling of the $\mathrm{GeV}$ data provides support for a maximum lobe age of $\sim 8 \times 10^{7} \mathrm{yr}$.

Figure 2 shows a SED representation for an epoch time $t_{\max }=8 \times 10^{7} \mathrm{yr}$ (for the SED for $t=10^{7} \mathrm{yr}$ see Ref. 8), with a maximum electron Lorentz factor $\gamma_{\max }=2.5 \times 10^{6}$ and $1.5 \times 10^{6}$ for the north lobe and the south lobe, respectively. Note that in this case the contribution by inverse-Compton scattering of CMB photons alone would be sufficient to account for the observed HE spectrum (see the

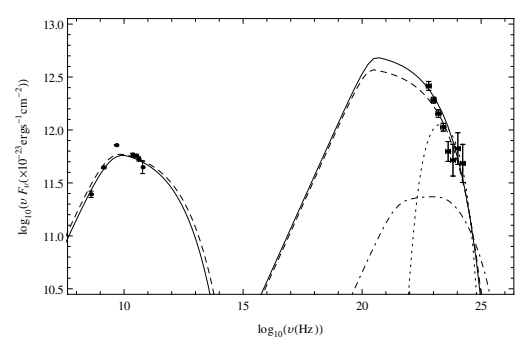

(a)

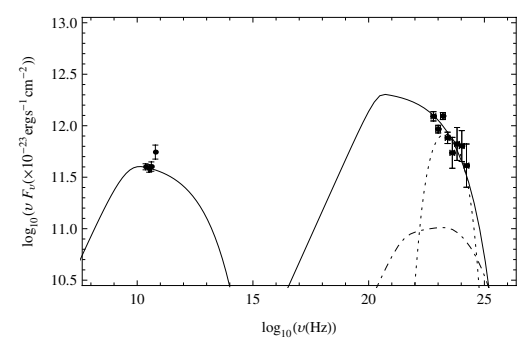

(b)

Fig. 2. SED for $t=8 \times 10^{7} \mathrm{yr}$ and for a mean magnetic field value $B$ for the south lobe and the $\gamma$-ray excess region in the north lobe of $0.91 \mu G$ and $1.17 \mu G$, respectively. The dot-dashed line in (a) represents the IC contribution due to EBL upscattering while the dashed line shows the result for $t=10^{8} \mathrm{yr}$. The possible $\gamma$-ray flux expected from pp-interactions given a thermal gas density $n=10^{-4} \mathrm{~cm}^{-3}$ is also shown (dotted line). 
solid line in Fig. 2). On the other hand, for an epoch time $t$ exceeding $t_{\max }=8 \times 10^{7}$ yr, the high-energy part of the SED would no longer be consistent with the data (see the dashed line in Fig. 2 for $t=10^{8} \mathrm{yr}$ ). These considerations provide additional support for a finite age $<10^{8} \mathrm{yr}$ of the lobes. The maximum total energy of electrons in both lobes is found to be $\approx 6 \times 10^{57} \mathrm{erg}$, with the total energy in particles and fields comparable to the $10^{7} \mathrm{yr}$-case, thus requiring only a relatively modest mean kinetic jet power of $\sim 10^{43} \mathrm{erg} / \mathrm{s}$.

In principle, a hadronic origin is conceivable as well: Once protons are efficiently injected, they are likely to remain energetic since the cooling time for pp-interactions is $t_{\mathrm{pp}} \approx 10^{15}\left(\mathrm{n} / 1 \mathrm{~cm}^{-3}\right)^{-1} \mathrm{~s}$. High-energy protons interacting with the ambient low-density plasma can then produce neutral pions which decays into two $\gamma$-rays. Similar to before, we employed a power-law proton distribution with an exponential cut-off to model the SED. Furthermore, a thermal plasma density $n=10^{-4} \mathrm{~cm}^{-3}$ for the giant radio lobes of Cen $\mathrm{A}$ has been assumed. This model representation is depicted by a dotted line in Fig. 2. In both lobes, the power-law index of the proton population is $\alpha=2.1$, and the high-energy cut-off is $E_{\max } \simeq 55 \mathrm{GeV}$. The maximum total energy $W_{p}$ is proportional to the gas number density $n$, so that $W_{p} \simeq 10^{61}\left(n / 10^{-4} \mathrm{~cm}^{-3}\right)^{-1} \mathrm{erg}$, obtained here, should be considered as an upper limit.

\section{Conclusions}

We have highlighted recent results from $\mathrm{HE}$ observation of the radio galaxy Cen $\mathrm{A}$. Accordingly, the core of Cen A shows a more complex spectral gamma-ray behavior than previously anticipated ("unusual" break at $4 \mathrm{GeV}$ ), while the HE $\gamma$-ray emission from the lobes do not fully coincide with the radio lobes (e.g., excess region in the north lobe). These results indicate that Cen A is a promising target for studies with the next generation HE/VHE $\gamma$-ray instruments (e.g. Cherenkov Telescope Array).

\section{References}

1. G. Harris, M. Rejkuba and W. Harris, PASA 27, 457 (2010).

2. B. Fanaroff and J. Riley, MNRAS 167, 31P (1974).

3. R. Morganti, R. Fosbury, R. Hook et al., MNRAS 256, 1P (1992).

4. M. Chiaberge, A. Capetti and A. Celotti, MNRAS 324, L33 (2001).

5. F. Israel, A\& A Rev. 8, 237 (1998).

6. A. Abdo et al. (Fermi-LAT), Science 328, 725 (2010a).

7. A. Abdo et al. (Fermi-LAT), ApJ 719, 1433 (2010b).

8. R. Yang, N. Sahakyan, et al., A\&4 542, A19 (2012).

9. N. Sahakyan, R. Yang, et al., ApJL 770L, 6S (2013).

10. F. Aharonian et al., (H.E.S.S. Collaboration), ApJ 695, L40 (2009).

11. F. Rieger, IJMPD 20, 1547 (2011).

12. M. Hardcastle and J. Croston, MNRAS 415, 133 (2011).

13. H. Alvarez, et al., A $\& A$ 355, 863 (2000).

14. M. Hardcastle, C. Cheung, I. Feain and L. Stawarz, MNRAS 393, 104 (2009). 